Transcontinentales

continentales

Sociétés, idéologies, système mondial

\title{
La nouvelle donne du logement à Shanghai
}

Transition économique et changement social

\section{Valérie Laurans}

\section{CpenEdition}

\section{Journals}

Édition électronique

URL : http://journals.openedition.org/transcontinentales/527

DOI : $10.4000 /$ transcontinentales.527

ISBN : 978-2-8218-1112-6

ISSN : 1775-397X

Éditeur

Editions de la maison des sciences de l'homme

\section{Édition imprimée}

Date de publication : 31 décembre 2006

Pagination : $31-46$

ISBN : 978-2-200-92170-5

ISSN : 1950-1684

\section{Référence électronique}

Valérie Laurans, «La nouvelle donne du logement à Shanghai », Transcontinentales [En ligne], 3 | 2006, document 3, mis en ligne le 12 octobre 2011, consulté le 08 septembre 2020. URL : http://

journals.openedition.org/transcontinentales/527 ; DOI : https://doi.org/10.4000/transcontinentales. 527

Ce document a été généré automatiquement le 8 septembre 2020.

Tous droits réservés 


\title{
La nouvelle donne du logement à Shanghai
}

Transition économique et changement social

\author{
Valérie Laurans
}

1 Métropole moderne ayant atteint son apogée durant l'entre-deux-guerres, Shanghai fut bâtie sur la finance et le béton. En 1949, l'avènement de la République populaire de Chine sonna le glas de cette éblouissante ascension. Victime du communisme, la ville trépidante fut condamnée à sombrer dans le sommeil pour quatre décennies. Vilipendée par le nouveau système, la promotion immobilière fut anéantie et la construction resta insuffisante faute de moyens jusqu'aux années 1980.

2 Or les bouleversements qui ont affecté Shanghai depuis le début des années 1990 se sont effectués avec une rapidité étourdissante. Aucune métaphore- réveil, redéploiement, mutation, métamorphose, renaissance - n'en donne l'exacte mesure. La revitalisation en cours ne possède tout simplement pas d'équivalent au monde... Son aspect le plus frappant réside sans doute dans la reprise du développement immobilier et le rythme effréné de la construction. Le remodelage du visage urbain qui en résulte n'en constitue pourtant pas la conséquence majeure. Le passage de l'économie planifiée à l'économie de marché a avant tout rendu possible le renouvellement du parc résidentiel du premier pôle urbain de Chine. L'importance de ce phénomène est aujourd'hui déterminante. En 2004, le secteur du bâtiment atteignait 62,25 milliards de $\mathrm{RMB}^{1}$ et occupait à lui seul $12 \%$ du PIB shanghaien (soit 745 milliards de RMB). Affichant une progression de $20 \%$ par rapport à l'année 2003, ce niveau couronnait une hausse rapide impulsée à la fin des années 1980.

3 Si cette croissance s'est révélée fructueuse d'un point de vue économique, elle a par ailleurs vivement affecté la société. Visant dans une large mesure à pallier le désinvestissement de l'État du secteur du logement, le développement de l'immobilier s'est accompagné d'une reconfiguration administrative de la cité et génère actuellement une dynamique de ségrégation spatiale exacerbée. Bien que les effectifs de la population shanghaienne soient officiellement restés stables au cours des quinze dernières années ${ }^{2}$, la reprise de la construction s'est inscrite dans un contexte de 
pénurie d'habitations endémique. On assiste ainsi à un étalement urbain doublé d'un déplacement des citadins à faibles revenus vers la périphérie.

4 Afin d'éclairer la nouvelle donne du logement à Shanghai, le présent article se propose de faire le point sur la transition économique et sur le changement social qui accompagnent ce mouvement. Dans ce but, il précisera d'abord les spécificités de la reprise de la construction shanghaienne à partir des années 1990. Il récapitulera ensuite les principales étapes du passage de l'immobilier résidentiel à l'économie de marché de 1980 à 2005. Il abordera enfin les conséquences sociales de l'actuelle explosion du marché du logement.

\section{Le renouveau de la construction immobilière à Shanghai}

5 Avec la venue de Deng Xiaoping au pouvoir en 1978, la Chine entre dans une ère de réformes économiques. Ce changement de contexte politique favorise la reconstruction du secteur de l'immobilier shanghaien.

\section{Un changement de contexte politique}

6 Au début des années 1980, les potentialités de la plus grande cité de Chine ne sont plus exploitées depuis trente ans. Les impératifs de la libéralisation économique du pays imposent cependant de reconsidérer le statut de la ville. Après avoir été dénigrée durant la période maoïste, elle redevient un espace voué à la consommation, tandis que son port de commerce doit recouvrer une dimension internationale. Le schéma directeur établi en 1986 répond à ce virement de cap politique initié par le gouvernement central. Shanghai peut désormais espérer prendre un nouvel essor, mais le poids des charges fiscales prélevées par l'État et la politique timorée de ses dirigeants entravent encore ses mouvements ${ }^{3}$.

7 Ce n'est qu'au début des années 1990, grâce à l'accélération de la politique de réformes, que la ville trouve les moyens de remanier son système de gestion urbaine en profondeur. Le développement spectaculaire de Shanghai durant les années 1990 tient ainsi à deux facteurs. La création puis la décision de la mise en valeur, par le gouvernement central, de l'arrondissement de Pudong va d'abord drainer une importante manne financière composée de fonds publics et de capitaux étrangers. La réforme du droit d'utilisation du sol permet parallèlement -l'ouverture d'un marché immobilier fleurissant et laisse augurer de substantiels profits ${ }^{4}$. Le redéploiement du parc immobilier shanghaien s'inscrit dans le cadre d'un processus de longue durée enclenché depuis le début des années 1980. Il s'agit de la reconversion du secteur du logement à l'économie de marché. Validé lors de l'approbation du 8• plan quinquennal en 1991, l'élargissement progressif du marché de l'habitat soutiendra la croissance économique à partir du 9c plan quinquennal (1996-2000). L'explosion du marché du logement, favorisée par les directives du 10॰ plan quinquennal (2001-2005), suscitera ensuite l'expansion d'une bulle spéculative. Avant de préciser la chronologie de ce basculement puis d'en aborder les conséquences, apprécions à travers quelques chiffres les caractéristiques du spectaculaire redéploiement immobilier des quinze dernières années. 


\section{La reprise de la construction de 1991 à 2005}

8 Le nombre de tours construites depuis le début des années 1990 permet d'emblée d'évaluer l'ampleur de la fabuleuse évolution du parc immobilier shanghaien. De 1991 à 2000, sur l'ensemble du territoire de la municipalité de Shanghai, ont été achevés non moins de 3400 bâtiments d'une hauteur de plus de 28 mètres (10 étages) et 186 édifices de plus de 100 mètres (33 étages). Leur construction s'est opérée au rythme d'un étage achevé tous les trois jours permettant la livraison d'un immeuble par semaine ${ }^{5}$... Bien que les architectures les plus impressionnantes telles que la tour Jinmao achevée en 1998 (88 étages culminant à 492 mètres de hauteur) hébergent centres commerciaux, bureaux et hôtels de luxe, le renouveau de la construction durant les années 1990 concerne principalement le parc résidentiel shanghaien.

9 Au printemps 1992, lors de sa tournée dans le Sud (nanxun), Deng Xiaoping encourage l'édification de Shanghai et insiste particulièrement sur le redéploiement des ensembles d'habitations. À Shanghai, les insuffisances du parc immobilier résidentiel, vétuste et surpeuplé, imposent des choix qui n'ont que trop tardé. L'exiguïté des habitations constitue un problème endémique auquel le pouvoir communiste n'a accordé qu'une importance relative durant les quarante premières années du régime. Au début des années 1980, la ville évoquait plus que jamais l'image de la promiscuité ${ }^{6}$. À partir du début des années 1990, la construction massive d'habitations neuves va rapidement estomper ce cliché. De 1993 à 2004, 169 millions de mètres carrés de logements sont ainsi édifiés. Durant cette période, la superficie habitable s'élève de 6,7 à 14,8 mètres carrés per capita. La construction du parc résidentiel s'effectue actuellement à la cadence de plus de 300000 habitations livrées par an sur le marché ${ }^{7}$.

10 Si les chiffres de la production de grande envergure du logement sont en eux-mêmes impressionnants, il est également important de noter que depuis les balbutiements de ce redéploiement jusqu'à aujourd'hui (soit de 1986 à 2004), plus de $85 \%$ des bâtiments construits afin d'être placés sur le marché sont des immeubles d'habitations. Alors qu'en 1995 l'édification du logement n'absorbait que $60 \%$ des investissements placés dans la construction immobilière (toutes catégories de bâti confondues: habitations, bureaux, commerces, et autres), ce rapport a évolué pour atteindre $77 \%$ en $2004^{8}$. Le formidable renouveau du secteur de l'immobilier résidentiel à Shanghai s'est ainsi effectué pas à pas. On peut le décomposer en quatre grandes étapes.

\section{0 à 2005 : le passage du secteur de l'immobilier résidentiel à l'économie de marché}

11 Au tournant des années 1990, l'ouverture du marché du sol permet d'entreprendre, d'une part, le remaniement en profondeur du plan d'aménagement de Pudong ${ }^{9}$, tout en autorisant, d'autre part, la modernisation des infrastructures urbaines à Puxi. Cette mesure fondatrice a été préparée en amont et constitue une étape intermédiaire de la politique de réformes lancée par Deng Xiaoping. Depuis le début des années 1980, les bases d'un secteur de l'immobilier privé ont été progressivement posées à Shanghai. Cette refonte engageait tout d'abord la formation de promoteurs shanghaiens. L'ouverture du marché du sol permit ensuite de construire et de vendre une catégorie spécifique de logements réservés aux ressortissants de nationalité étrangère (chinois 
d'outre-mer et investisseurs occidentaux). Ce n'est qu'à partir du milieu des années 1990, que l'élargissement du marché de l'immobilier englobera l'ensemble du parc résidentiel de la ville.

\section{L'ouverture du marché foncier}

En République populaire de Chine, le sol est propriété de l'État qui gère seul son utilisation depuis les années 1950. Destinée à relancer l'économie, la réforme de ce monopole étatique ouvre le champ à la privatisation du secteur de l'immobilier. Cette mesure, appliquée de façon expérimentale depuis 1988 sur le territoire de Puxi ${ }^{10}$ centre historique de Shanghai - autorise l'État à céder les droits d'utilisation du sol à des investisseurs privés.

À partir de décembre 1987, plusieurs parcelles situées à proximité de l'aéroport international de Hongqiao sont ainsi louées de façon expérimentale à des joint venture ou à des entreprises de Hong Kong. Cet essai fait suite à une expérience développée à Shenzhen ${ }^{11}$ depuis 1985, qui s'inspire elle-même directement du modèle de gestion du sol hongkongais. Il sera progressivement généralisé à l'ensemble du pays. Par la suite, les parcelles pouvant être cédées à des investisseurs étrangers (principalement de Hong Kong, Taiwan et Singapour) se concentrent à l'intérieur du périmètre des zones de développement ${ }^{12}$. Ce périmètre s'étendra graduellement à l'ensemble de la ville. Les premiers édifices construits pour être placés sur le -marché sont des tours de bureaux, des complexes commerciaux, des hôtels, des «logements destinés aux résidents étrangers" (waixiaofang). Cette phase annonce le développement du logement à venir. Celui-ci a par ailleurs été préparé depuis une dizaine d'années par la formation de professionnels de la construction. 


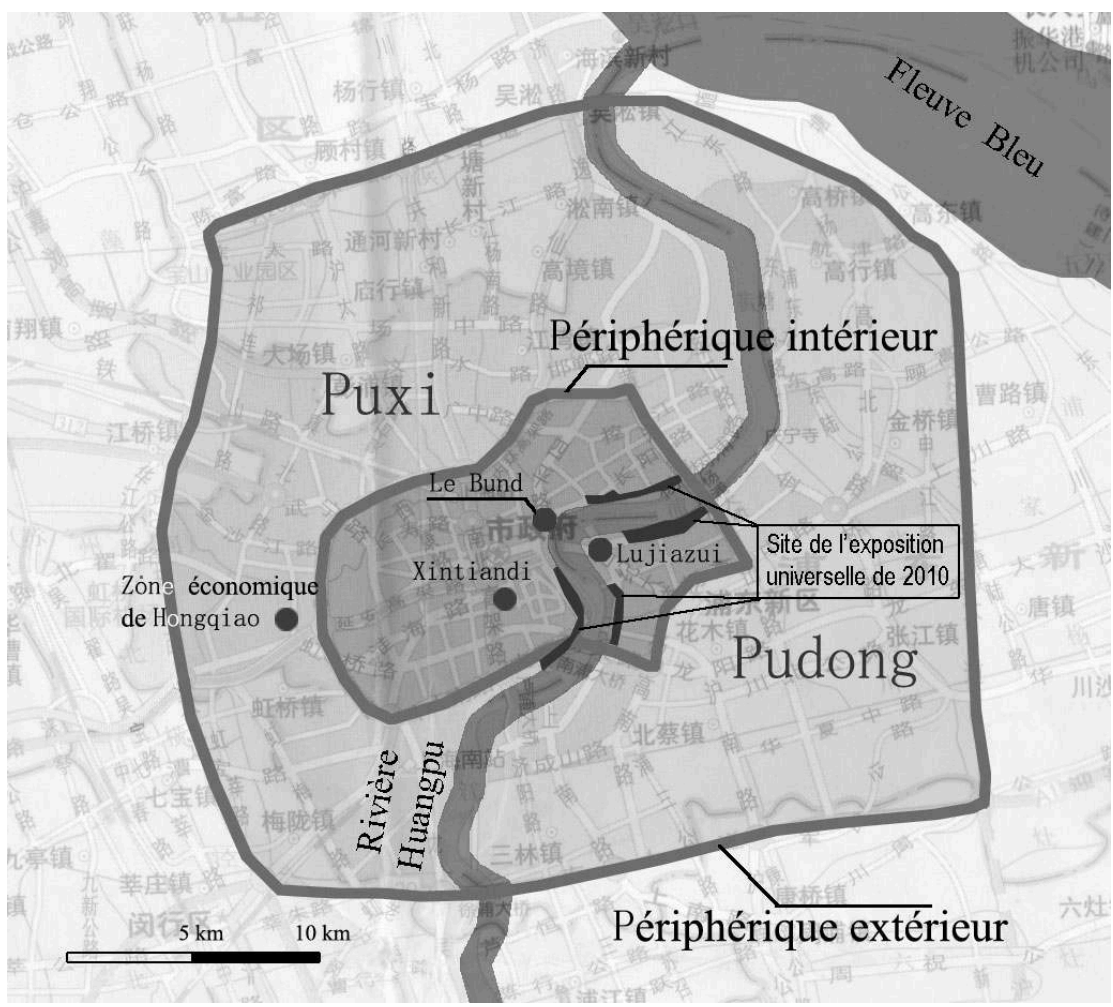

PÉRIPHÉRIQUE INTÉRIEUR - Il délimite le noyau historique de Shanghai et encercle le parc résidentiel de la ville édifié avant les années 1980. Ce territoire est en cours de réhabilitation et fait l'objet d'une profonde reconfiguration sociale.

PÉRIPHÉRIQUe EXTÉRIEUR - Le schéma directeur de Shanghai 2000-2020 redéfinit le périmètre du centre-ville. Cette mesure vise à limiter dans l'espace l'expansion du parc des logements neufs destinés à être placés sur le marché.

zone ÉCONOMIQUe DE HONGaIAO - Site expérimental sur lequel se situent les premières parcelles cédées à des investisseurs privés dans le cadre de l'ouverture du marché du foncier à la fin des années 1980.

LUJIAZUI- Centre bancaire et financier de Shanghai. Ce site fait face aux prestigieux édifices du Bund à Puxi. Il incarne à la fois l'essor de Pudong, le renouveau de la métropole au tournant du xxi siècle, et la vitrine de la Chine moderne sur le monde. Durant les années 1990, son édification a vivement stimulé le processus de reconversion du secteur de l'immobilier à l'économie de marché.

XINTIANDI- Il s'agit du lilong (lotissement spéculatif typique de l'habitat du Shanghai des concessions) au sein duquel s'est tenu le premier congrès du Parti communiste chinois en 1921. Restauré en 1999 et reconverti en site touristique dédié aux loisirs et à la consommation de luxe, ce lieu de mémoire s'insère au cœur d'un vaste projet de réhabilitation urbaine.

SITE DE L'EXPOSITION UNIVERSELLE DE 2010 - Son implantation le long des berges de la rivière Huangpu et au sud de l'ancienne ville chinoise entraîne actuellement l'accélération du processus de renovation urbaine en centre ville.

\section{La formation de la promotion immobilière}

À Shanghai, la promotion immobilière renaît dès 1979 sous l'impulsion de la "politique des qiaohuifang ». L'édification de ce parc résidentiel distinct des logements ordinaires, destiné aux ressortissants étrangers issus de la diaspora chinoise, vise à encourager le retour et l'implantation des Chinois d'outre-mer sur le continent. Deux sociétés gérées par des organismes municipaux sont alors créées afin d'en assurer la construction. Il faut cependant attendre une dizaine d'années supplémentaires pour que ce secteur 
d'activité commence à se détacher de l'état. La première société de promotion immobilière par actions voit alors le jour en 1988.

La réforme administrative de 1992, qui interdit aux administrations de se lancer dans les affaires, marque une étape décisive de la transition de l'économie planifiée vers l'économie de marché. Avant cette date, toutes les sociétés de promotion immobilière étaient étroitement liées à l'administration, chaque municipalité d'arrondissement ayant édifié un bureau spécifique destiné à la construction des qiaohuifang. À partir de 1992, les contraintes se relâchent et les exigences pour créer une entreprise de promotion immobilière sont plus faibles. Une habilitation est délivrée à toute société désirant se lancer dans la construction, sous réserve d'un apport de capitaux minimum et d'une garantie de compétences techniques ${ }^{13}$. En juillet 1995, les promoteurs immobiliers chinois entrent de plain-pied sur le marché de la construction du logement. Le gouvernement de Shanghai entérine un document permettant aux administrations locales ${ }^{14}$ de céder leurs droits d'utilisation du sol (l'État en restant propriétaire). Les entreprises privées de développement et de promotion immobilière sont dès lors autorisées à lotir un terrain et à vendre les immeubles qui y sont construits. Les sociétés immobilières chinoises, qui jusqu'à présent étaient dépendantes d'une association avec une entreprise étrangère, gagnent une nouvelle fois en autonomie.

\section{La privatisation du marché du logement}

16 Tandis que l'État entérine l'ouverture du marché du sol dans le périmètre de la zone de développement de Hongqiao en 1988, la "politique des waixiaofang" concernant plus particulièrement le logement est lancée. Cette mesure visant les ressortissants non chinois permet l'ouverture d'un micro-marché du logement neuf. Bien qu'encore majoritairement réservé aux étrangers, ce marché est progressivement ouvert aux acquéreurs particuliers de nationalité chinoise. Parallèlement, les unités de travail ${ }^{15}$ sont autorisées à solder de façon expérimentale une partie de leur parc résidentiel. La réforme du logement qui prend effet en mai $1991{ }^{16}$ précède alors la privatisation du marché de l'immobilier résidentiel destiné aux ressortissants chinois.

17 En 1992, l'État poursuit la vente de son parc d'habitations. Celle-ci s'intensifie de 1994 à 1995. Au cours de cette phase débute également la production de masse de logements neufs placés sur le marché. En 1996, la "politique du hukou bleu» encourage les résidents non shanghaiens à acquérir une habitation à Shanghai. Cette mesure constitue une première extension nationale du marché du logement shanghaien.

La même année, le bureau du développement du logement est créé. Ce nouvel organisme dépend directement du département de la construction de la municipalité de Shanghai. Il va permettre d'encadrer et de réguler la construction immobilière. Alors que l'attribution du logement par les unités de travail prend fin à partir de 1998, des politiques d'emprunt et d'exonération fiscale se mettent en place pour inciter les résidents shanghaiens à investir dans l'immobilier résidentiel ${ }^{17}$.

L'investissement dans la pierre prend alors le relais des placements en bourse dont les cours se sont effondrés sous l'effet de la crise asiatique en 1997. La perte de confiance dans cette institution financière est d'autant plus grande que la variation des valeurs boursières est largement maîtrisée par l'État. Le marché du logement sera désormais 
soumis à une dérégulation partielle tandis que l'immobilier résidentiel fera l'objet d'une importante spéculation.

\section{0 à 2005 : l'expansion du marché immobilier}

20 Avec l'envol de la production des logements placés sur le marché, une première phase spéculative démarre entre 1994 et 1995 . Elle retombe cependant brutalement à partir de 1997 en raison de la crise asiatique. Durant la même année, une politique d'encadrement et des contre-mesures de régulation de la construction interviennent afin d'accompagner la baisse des ventes et de limiter le stock d'habitations invendues ${ }^{18}$. L'augmentation des ventes de logements ne reprend timidement qu'à partir de 2000. Cette relance peut-être imputée à l'arrêt définitif de la politique d'attribution du logement par les unités de travail. Elle est également encouragée par l'entrée de la Chine dans l'OMC: en août 2001, la distinction entre marchés de l'immobilier résidentiel pour étrangers et pour Chinois est abolie ${ }^{19}$. La construction de logements augmente timidement sans pour autant atteindre son niveau culminant de 1997.

21 Avec l'annonce de la sélection de Shanghai comme site officiel de l'exposition universelle de 2010, l'évolution du marché du logement prend un nouveau tournant. En 2002, la restriction qui touchait les investissements étrangers dans la construction et la rénovation du parc immobilier shanghaien est supprimée. Le marché de l'immobilier résidentiel à Shanghai s'ouvre au monde. Son développement devient exemplaire pour l'ensemble du pays. Une deuxième phase de surchauffe débute alors. En un an, le nombre des logements placés sur le marché augmente de $70 \%$. Les prix de l'immobilier s'envolent ${ }^{20}$. Les spéculateurs d'outre-mer et de l'ensemble de la Chine continentale investissent volontiers dans l'immobilier shanghaien pour accélérer la modernisation de la métropole. Ses résidents mettent également à profit les nouvelles possibilités d'emprunt bancaire visant à encourager l'acquisition d'un logement neuf.

Fin 2003, le bureau de développement du logement est dissout. La production d'habitations neuves - que cet organisme avait pour objet de promouvoir - est officiellement jugée suffisante. En réalité, les parcelles à construire en centre-ville et en proche périphérie se raréfient. Il s'agit désormais d'encourager la rénovation du parc immobilier ancien. Des directives sont prises pour limiter la spéculation foncière : le 31 août 2004 le marché du sol devient en théorie transparent. En mai 2005, une série de mesures décrétées au niveau national s'attaque à l'expansion trop rapide de la bulle spéculative dans les villes côtières chinoises ${ }^{21}$. Shanghai est tout particulièrement visée, le développement de son marché immobilier doit ralentir sa croissance. L'accent est désormais mis sur la construction d'un parc résidentiel de standard moyen accessible à l'ensemble de la population. Les prix du logement stagnent mais ne chutent cependant pas. L'évolution incertaine des cours de l'immobilier laisse les acheteurs potentiels dans l'expectative.

En 2005, alors que la croissance de la ville s'appuie plus que jamais sur les profits dégagés par le développement du secteur de l'immobilier, il s'agit de freiner l'essor du marché du logement. Les dirigeants shanghaiens voient en effet une menace pour la stabilité sociale dans la croissance excessive des prix du logement liée à la spéculation immobilière. Le gouvernement central dirigé depuis 2003 par Hu Jintao est quant à lui soucieux de rééquilibrer le réseau métropolitain chinois et cherche à réduire l'écart qui 
se creuse entre Shanghai et Pékin. Au terme d'un quart de siècle de réformes, le déploiement exponentiel de l'immobilier devient difficilement maîtrisable. 
Tableau 1 


\section{Repèreshistoriques}

1978Arrivée de Deng Xiaoping au pouvoir, lancement de la politique de réformes économiques.

1987 Crise asiatique.

2001 Entrée de la Chine dans l'OMC.

2002 Annonce de la désignation officielle de Shanghai comme site de l'exposition universelle de 2010.

2005 Le gouvernement formé par Hu Jintao tente de rééquilibrer l'écart creusé entre Pékin et Shanghai.

\section{La formation de la promotion immobilière à Shanghai}

1979Lancement de la «politique des qiaohuifang»: construction de logements destinés à l'implantation des chinois d'outremer sur le continent.

1980 L'idée de la réforme du logement est lancée. Les premières sociétés de promotion immobilière sont créées.

1983 Les règles du marché sont introduites dans la construction du logement.

1987Début de la «reconversion de l'utilisation du sol ».

1988 La première société de promotion immobilière par actions voit le jour.

1990 Quatre-vingts sociétés de promotion immobilière sont recensées à Shanghai.

1992 Les contraintes pour créer une société de promotion immobilière sont assouplies, leur nombre est aussitôt décuplé.

1995 Les promoteurs immobiliers chinois ont la possibilité d'acquérir un droit d'utilisation du sol à durée limitée leur permettant de lotir un terrain et de vendre les logements qui y Transcontinentales 3 Sont COnstruits
.

1996 Le gouvernement central décrète que «le secteur de l'immobilier résidentiel constitue un pilier de marché sont édifiées dans la zone économique de Hongqiao.

1991 La « réforme du système du logement à Shanghai » est lancée, elle va s'étaler sur une dizaine d'années.

1993 L'État met en vente une partie de son parc immobilier de façon expérimentale.

1994- Intensification de la vente du parc résidentiel

1995appartenant à l'État. Début de la production de masse de logements neufs destinés à être placés sur le marché.

1996 Lancement de la «politique du hukou bleu »: cette mesure correspond à une extension nationale du marché du logement.

1998- Fin de l'attribution du logement par les unités

2000de travail.

2001 Fin de la séparation des marchés du logement pour Chinois et pour étrangers extension internationale du marché du logement shanghaien.

2002 La restriction des investissements étrangers dans la construction et la rénovation du parc immobilier shanghaien est supprimée. Le marché de l'immobilier résidentiel à Shanghai s'ouvre au monde.

\section{Bulle spéculative : croissance et régulation}

1994- Première phase de surchauffe spéculative du

1995marché de l'immobilier résidentiel à Shanghai.

1996 Création du bureau du développement du logement: organisme placé sous la direction du Département de la construction, il vise à encadrer et à réguler la construction immobilière et fixe pour ce faire les normes de conception et de construction des habitations.

2002 Début d'une deuxième phase de surchauffe du marché du logement.

2003 Dissolution du bureau du logement, le nouveau parc résidentiel étant jugé suffisant.

2003 Les gouvernements d'arrondissements réfléchissent aux moyens de réhabiliter leur parc résidentiel hérité de l'époque maoïste. 


\section{Les conséquences sociales de l'explosion du marché du logement}

Dans la perspective de l'exposition universelle de 2010, l'extension du marché du logement fournit à la municipalité de Shanghai l'occasion et les moyens d'accélérer le processus de rénovation urbaine. Le gouvernement local cherche de la sorte des solutions pour financer la réhabilitation du parc immobilier ancien tout en offrant de nouvelles perspectives d'investissement aux promoteurs immobiliers. Ce processus a une incidence directe sur l'organisation du territoire urbain. La reconfiguration administrative qui s'ensuit se double d'une exacerbation des phénomènes de ségrégation spatiale. Tandis que le déploiement du parc de logements neufs en périphérie entraîne un changement d'échelle de la cité, la réhabilitation du centre urbain génère d'importants mouvements de population. La nouvelle donne du logement à Shanghai s'accompagne dès lors de conflits sociaux récurrents.

\section{La reconfiguration administrative de la cité}

$25 \mathrm{Au}$ tournant du XXI siècle, le schéma directeur 2000-2020 ${ }^{22}$ fixe les directions de développement envisagées par la commission de la planification urbaine. Celles-ci ne présentent pas de grandes nouveautés par rapport au précédent plan d'urbanisme qui datait de 1986. L'ambition qui les sous-tend est de permettre à Shanghai de reconquérir un statut de métropole internationale. Il convient donc de transformer la ville industrielle en une place financière et économique de première importance. À cette fin, la métropole tente de présenter le visage d'une ville propre et mène plusieurs opérations cosmétiques: de la dépollution de ses cours d'eau au développement des espaces verts, en passant par la mise en scène de son patrimoine historique.

D'un point de vue structurel, les directives du nouveau schéma directeur se traduisent par un réajustement de l'échelle urbaine. Alors que l'intégration de Pudong est renforcée, Puxi poursuit une double politique de modernisation et de désengorgement. Les industries sont déplacées en dehors du périphérique extérieur, leur présence est relayée par celle d'immeubles de bureau, de centres commerciaux, de résidences de standing, etc. L'implantation des nouvelles zones de logements de masse s'effectue quant à elle en dehors de la ceinture autoroutière intérieure ${ }^{23}$. La redéfinition du périmètre de la ville, désormais délimitée par la ceinture autoroutière extérieure, vise à les assimiler au centre urbain. Afin de promouvoir ce mouvement et de faciliter l'intensification des migrations biquotidiennes qui en résulte, les infrastructures de transport en commun (métro et tramway aérien) font l'objet d'un développement soutenu.

À la fin de l'année 2002, l'élection de Shanghai comme site de l'exposition universelle de 2010 suscite le remaniement de ce plan validé par le Comité central du PCC depuis à peine un an. Le redimensionnement de la ville se poursuit ainsi en banlieue. Dans la perspective de 2010, les projets de réaménagement du centre se font par ailleurs plus audacieux. Le site destiné à accueillir l'exposition universelle s'implante au cœur du noyau historique et mettra en valeur la relation qui unit la ville à son fleuve. Son 
édification impose l'éviction des populations qui y résident. La rénovation des quartiers anciens situés à proximité immédiate de cet épicentre, telle la vieille ville chinoise, doit également être accélérée. Les opérations de destruction et de renouvellement urbain s'étendent enfin tout au long de la façade nord du fleuve Huangpu.

En termes de marketing urbain, l'image de modernité véhiculée par Pudong ne suffit plus à affirmer la spécificité de Shanghai au regard des autres villes mondiales. La métropole se tourne donc vers le patrimoine architectural hérité de l'époque des concessions afin de proclamer sa propre identité. Le quartier de Xintiandi dont les travaux de rénovation avaient débuté dès 1999 devient la carte de visite de la ville (jusqu'en 2002, cette fonction était assurée par la façade architecturale de Lujiazui à Pudong: vitrine de la Chine moderne sur le monde). En 2004, un plan de préservation visant douze secteurs historiques est élaboré. Ces lieux épargnés par les démolitions dues à la montée de la pression immobilière pourront à terme accueillir un réseau de circuits touristiques.

La préparation de l'exposition universelle de 2010 ménage de vastes espaces de développement au secteur immobilier shanghaien. Ce faisant, elle entraîne une reconversion massive des quartiers d'habitations populaires du centre-ville. La construction de grande envergure du logement neuf occasionne par ailleurs un étalement des zones résidentielles en périphérie urbaine. La conjuguaison de ces deux phénomènes aboutit à une nouvelle répartition des différentes catégories de résidents urbains.

\section{Une ségrégation spatiale exacerbée}

30 Marquée par le passage du secteur de l'immobilier résidentiel à l'économie de marché, la reprise de la construction à Shanghai modifie radicalement les règles d'accès à l'habitat. Jusqu'à la fin du XX⿳亠口冋口 siècle, le logement était attribué par l'État. L'abandon du "système de bien-être " sur lequel reposait cette politique (fuli fen fang) se double d'une réévaluation des normes du confort domestique. Mais une part importante de la société ne possède ni le désir, ni les moyens de payer le coût d'un tel procès. Les habitations standard ${ }^{24}$ restent hors de portée des citadins qui ne présentent pas de revenus supérieurs à la moyenne ${ }^{25}$. La nouvelle donne du logement paraît donc a priori en leur défaveur. Selon les dirigeants chinois, l'investissement financier représenté par l'habitat ainsi que la jouissance de meilleures conditions de logements devraient pourtant contribuer à pérenniser la stabilité sociale.

Sur le terrain, on constate que le développement du marché de l'immobilier résidentiel se traduit par un grand "remue-ménage ", c'est-à-dire par une reconfiguration sociale de l'espace urbain sans précédent. De façon schématique, cette logique tend à disséminer la pauvreté dans des enclaves périphériques et à concentrer les richesses au cœur du centre-ville. Concernant les dix arrondissements composant ce territoire, 38 millions de mètres carrés de bâti ancien ont été détruits depuis 1990. Fait caractéristique du développement métropolitain, les opérations de relogement auraient affecté 3,5 à 4 millions d'habitants depuis 1992. Ces résidents participent de façon contrainte à la gentrification des espaces les plus convoités. Selon certains économistes chinois, ce phénomène constituerait aujourd'hui «l'œil du cyclone » du secteur de la promotion immobilière. Enjeu économique, les déplacements sont aussi la source d'affrontements retentissants entre administrateurs et administrés. Certains 
conflits significatifs tels que l'affaire Zhou Zhengyi ${ }^{26}$ ont fait l'objet d'une importante couverture dans la presse nationale et internationale. L'affaire Maiqili ${ }^{27}$ a été moins largement divulguée dans les médias. La condamnation exemplaire des crimes perpétrés par les responsables de la société de déplacement du groupe Chengkai ${ }^{28} \mathrm{a}$ pourtant servi les autorités shanghaiennes. Tandis que le gouvernement encourage la croissance rapide du secteur de l'immobilier, la propagande officielle prétend lutter contre ses fréquents dérapages.

Han Zheng, l'actuel maire de Shanghai aurait ainsi déclaré que la multiplication des déplacements, mettant en péril la paix sociale, exerçait une influence néfaste sur le développement de la métropole. En termes purement économiques, l'intérêt des expulsions est pourtant indéniable. En effet, la dynamique suscitée par les évictions entretient la croissance artificielle des prix de l'immobilier ${ }^{29}$ et soutient le développement à venir de la cité ${ }^{30}$. Arbitre d'un rapport de forces inégales, les autorités de Shanghai relèvent dans ce contexte un défi stratégique. Dans le but de stimuler le secteur de la construction, elles font le pari de favoriser les acteurs économiques tout en s'efforçant de ménager les intérêts individuels de la majorité des résidents urbains. Face à cette évolution, les acteurs institutionnels et sociaux sont amenés à modifier leurs pratiques et leurs logiques d'action.

\section{L'évolution du jeu social}

Symptôme de l'accroissement des inégalités urbaines, la réhabilitation de l'habitat ancien s'illustre par des conflits de « dominance urbaine ${ }^{31}$ " (accaparement physique et symbolique de l'espace urbain au profit des détenteurs du pouvoir effectif). Les quartiers populaires du centre-ville constituent le vaste théâtre de ces affrontements. Le suivi du déplacement des habitants de Jianyeli ${ }^{32}$, quartier -d'habitation populaire situé au cœur de l'ancienne concession française, montre pourtant que la double volonté du gouvernement central et des pouvoirs locaux d'éviter les épreuves de force conduit à l'élargissement des marges de manœuvre individuelles. Si lors des opérations d'éviction ces épreuves ont bien lieu, elles -deviennent un moyen de négociation risqué mais qui peut s'avérer rémunérateur à la fois pour les représentants de la municipalité et pour les habitants.

Car les résidents concernés par un projet de déplacement possèdent rarement les moyens d'accéder à la propriété d'un logement neuf par le biais du marché. La finalité des expulsions est donc acceptée sous condition d'en marchander les modalités d'application. Concrètement les débats portent sur la base des compensations financières ou des dédommagements en nature ${ }^{33}$ attribués aux résidents déplacés. Cependant l'échange des logements libérés ne s'effectue pas selon des critères équitables. Les modalités en sont évaluées au cas par cas en fonction des négociations engagées entre société de déplacement et foyers déplacés. Dans de telles conditions, l'élargissement individuel de la marge d'action sur son propre destin ainsi que sur celui des siens prime sur l'édification d'une norme des compensations valable pour tous. La satisfaction ou le dépit de chacun est en fait relatif à l'amélioration subjective d'un cas particulier évalué à l'aune de son parcours de vie spécifique.

Du point de vue du pouvoir institutionnel, les négociations permettent de juguler les revendications des habitants insatisfaits dans le cadre de la nouvelle donne du logement à Shanghai. Ces accords de circonstance, bien que signés à grands frais, visent 
à garantir la paix sociale. Pour les citoyens ordinaires restés en marge du développement du confort à Shanghai, les opérations d'éviction fournissent l'occasion d'exiger de façon individuelle l'obtention de privilèges plus étendus. Ces avantages prennent la forme d'une augmentation substantielle des compensations (financières ou en nature) initialement prévues dans les contrats d'expulsion. L'organisation des contestataires insatisfaits, menacée de répression et synonyme de perte de tout avantage, reste cependant improbable. La constitution d'une société civile demeure en cela verrouillée.

\section{Conclusion}

À Shanghai, les trois plans quinquennaux qui se sont succédé de 1991 à 2005 ont soutenu le passage du secteur de l'immobilier résidentiel à l'économie de marché. Cette transition économique a engagé une nouvelle donne du logement et s'est traduite par une métamorphose physique et structurelle de la cité. Le nouveau déploiement du parc d'habitation constitue également une source de conflits entre administrateurs et administrés. Le mécontentement social engendré par la reconquête du centre-ville en fragilise les fondements, mais ne semble pas pour l'instant modifier en profondeur les rapports de force entre État et société civile.

L'élection de Shanghai comme site de l'exposition universelle de 2010 constitue aujourd'hui une échéance clé pour la métropole qui ambitionne de s'imposer dans le cercle restreint des pôles urbains d'importance mondiale. L'année 2006 correspond au début d'un nouveau plan quinquennal dont le terme sera couronné par la tenue de cet événement médiatique. Dans cette perspective, le gouvernement local accélère la modernisation des infrastructures urbaines. Il assure en cela, pour une décennie supplémentaire, la pérennité du développement de l'immobilier engagé durant les années 1990.

Si au-delà de cette échéance, il semble difficile d'anticiper sur les développements à venir du marché du logement, il est possible d'en interroger les orientations immédiates. La croissance accélérée du marché immobilier présente plusieurs menaces et inquiète les autorités shanghaiennes et le gouvernement central. À l'échelon local, la municipalité de Shanghai redoute les conséquences éco-nomiques et sociales de l'éclatement de la bulle spéculative entretenue par la dynamique des déplacements forcés. À l'échelon national, le gouvernement s'inquiète de l'impact des évictions massives sur la stabilité sociale. Il stigmatise également l'envol trop rapide du premier pôle économique de Chine. Il affiche enfin son désir de réduire l'écart creusé entre la métropole et les autres centres urbains chinois, notamment Pékin. La mise en application de mesures visant à combattre les -stratégies spéculatives ainsi que l'édification d'un encadrement juridique du développement urbain feront-elles d'une pierre deux coups ? Parviendront-elles à assainir le marché de l'immobilier chinois tout en contribuant à préserver les intérêts privés des résidents urbains? Quels ques soient les résultats des politiques locales et nationales, il est difficile d'imaginer à court terme une diminution significative des prix du logement. Le marché de l'immobilier résidentiel restera en cela inaccessible à une large demande populaire. Face à quoi, la marge de la société lésée par la nouvelle donne du logement choisira-t-elle de mobiliser significativement le droit en sa faveur? La poursuite des échanges d'avantages matériels devrait, en dernier ressort, décider du maintien du statu quo actuel. 


\section{BIBLIOGRAPHIE}

Sources françaises

- ANTIER G., « Le nouveau Shanghai. Ten-dances du développement en 2000 », rapport de mission, Municipalité de Shanghai, Région Île-de-France, février 2001, 26 p.

- BERGÈRE M.-C., Histoire de Shanghai, Paris, Fayard, 2002, 513 p.

- GED F., Shanghai. Portrait de ville, Paris, Institut français d'architecture, 2000, 64 p.

- GUIHEUX G., « Immobilier : les autorités à la fois juge et partie », Les Archives de l'actualité, CEFC

(Centre d'études français sur la Chine contemporaine), Hong Kong, juin 2005 ; version

électronique : http://www.cefc. com.hk/ fr/cefc/archives_actualite.

- HENRIOT C. et Z. ZHENG, Atlas de Shanghai. Espaces et représentations de 1849 à nos jours, Paris, CNRS

Éditions, 1999, 183 p.

- LAURANS V., « Logements et confort à Shanghai. L'exemple de wanli, ensemble résidentiel modèle ", Perspectives chinoises, $n^{\circ} 68$, CEFC (Centre d'études français sur la Chine contemporaine), Hong Kong, novembre-décembre 2001, p. 37-46.

- LAURANS V., "Shanghai : l'argument du confort pour déplacer les résidents urbains. Le cas de Jianyeli : projet-pilote de restauration ", Perspectives chinoises, $\mathrm{n}^{\circ} 87$, CEFC (Centre d'études français sur la Chine contemporaine), Hong Kong, janvier-février 2005, p. 10-21.

- NAGUY L., « Pudong ou Shanghai-Rive droite : nouvelle vitrine de la Chine ? », Perspectives chinoises, $\mathrm{n}^{\circ} 68$, CEFC (Centre d'études français sur la Chine contemporaine), Hong Kong, novembre-décembre 2001, p. 28-36.

- PIN A., « Le plan d'urbanisme de Shanghai 2000-2020 », rapport de stage, Consulat général de France à Shanghai, avril 2000, $31 \mathrm{p}$.

Sources chinoises

- CAI Y. et H. zHANG, Fangdi chan fazhan [Le développement de l'immobilier], Shanghai, Shanghai jingji fazhan congshu, Shanghai shehui kexueyuan chubanshe, 1999, 284 p.

- JIA G. et K. GUo, « Shanghai shi, tudi pizhun de shiben zuofa, ji guifan tu di de sikao » [Réflexions sur le marché et l'utilisation du sol à Shanghai], Zhongguo fangdi chan, Shanghai, 1998, p. 31-33.

- MAO J., « Gaige chuangxin - tuidong tuidong shanghai zhuzhai jianshi ke texu fazhan » [Réformer-imprimer un élan à la construction du logement à Shanghai et soutenir son développement], intervention lors du colloque Shanghai Housing Development, Shanghai, 15 et 16 novembre $2000,5 \mathrm{p}$.

- SHEN Z. et J. HUANG, Zhuzhai yu fangdi chan cidian [Dictionnaire de l'habitat et de l'immobilier], Shanghai, Xuelin chubanshe, 2001, 635 p.

- WANG A., « Shanghai zhuzhai fazhan jiancheng yu duici » [Évolution et contre-mesures du développement du logement à Shanghai], conférence donnée à l'occasion de la cession des Ateliers de Cergy-Pontoise à l'Université Tongji, Shanghai, 2 novembre 2000, 25 p.

- WANG W. et J. MAO, Shanghai 21 sheji chu de zhuzhai jianshi fazhan celüe [Stratégie de développement du logement au début du XXI siècle à Shanghai], Shanghai, Xuelin chubanshe, 2000. 
- Shanghai shi fangwu tudi ziyuan guanli ju, shanghai shi tongji ju, « Shanghai shi fangdi chan shicheng » [Le marché immobilier à Shanghai], Shanghai shehui kexueyuan chubanshe, années 2003-2004-2005.

- Shanghai shehui kexueyuan fangdi chan yanjiu zhongxin, Zhongguo fangdichan yanjiu [Études sur le marché immobilier], Shanghai, 2000, 159 p.

- Shanghai guihua ju, « Shanghai zhongti guihua 1986 » [Schéma directeur de la ville de Shanghai : actualisation de 1986].

- Shanghai guihua ju, « Shanghai zhongti guihua 2000-2020 » [Schéma directeur de la ville de Shanghai 2000-2020 : actualisation de 1999].

- Shanghai jianshe bianweihui, Shanghai jianshe 1996-2000 [La construction de Shan-ghai de 1996 à 2000], Shanghai, Shanghai shehui kexueyuan chubanshe, 2001, 794 p.

- Shanghai shi jianshe weiyuanhui, Shan-ghai jianshe tuji 1951-1996 [Atlas de la construction à Shanghai de 1956 à 1996], Shanghai, Shanghai kexue tishu wenxian chubanshe, 1998, 332 p.

- Shanghai shi zhuzhai fazhan ju, Fazhan zhong de shanghai zhuzhai [Le développement du logement à Shanghai], Shanghai, 1999.

-Shanghai shi zhuanzhi xilie congkan, Shanghai chengshi guihua zhi [Les annales de l'urbanisme de Shanghai], Shanghai, Shanghai shehui kexueyuan chubanshe, 1999, 702 p.

- Shanghai shi zhuanzhi xilie congkan, Shanghai fangdi chan guihua zhi [Les annales du secteur immobilier à Shanghai], Shanghai, Shanghai shehui kexueyuan chubanshe, 1999, 654 p.

\section{NOTES}

1. Dix RMB (renminbi) équivalent à un euro.

2. Le nombre de citadins possédant un permis de résidence (hukou) shanghaien était d'environ 13500000 en 2004. Ce chiffre ne présente qu'une très faible croissance par rapport à 1990 où la population de Shanghai était estimée à 13320000 habitants. L'augmentation de la population migrante (liudong renkou) représente en revanche une question délicate sur laquelle les statiques gouvernementales fournissent peu de précisions. Si ces résidents temporaires (en théorie) étaient estimés à 2000000 au début des années 1990 (voir les statistiques officielles publiées chaque année dans l'Almanach de Shanghai), ils sont néanmoins chiffrés à plus de 5000000 en 2003 par les médias chinois. Selon des sources non gouvernementales, la population totale de la cité pourrait ainsi actuellement avoisiner les 20000000 d'habitants répartis sur un territoire total de 6340 kilomètres carrés (superficie équivalente à celle d'un département français).

3. Concernant l'histoire et le renouveau de Shanghai, voir F. Ged, Shanghai. Portrait de ville, Institut français d'architecture, Paris, 2000, 64 p.

4. Voir F. Ged, op. cit.

5. Chiffres provenant du site www.NB119.com.

6. Au début des années 1980 , un résident shanghaien disposait d'une superficie habitable de moins de 4 mètres carrés.

7. Durant le $7^{\mathrm{e}}$ plan quinquennal (1986-1990) et la première étape du $8^{\mathrm{e}}$ plan quinquennal précédant l'intervention de Deng Xiaoping en faveur de l'accélération de la construction du logement en 1992 - on n'avait assisté qu'à une production totale de 47 millions de mètres carrés de logement.

8. Le total des investissements placés dans le secteur immobilier de 1986 à 2004 s'élève à 637 milliards 205 millions de RMB. 
9. Pour plus de précisions concernant les enjeux du développement de Pudong voir L. Naguy, «Pudong ou Shanghai-Rive droite : nouvelle vitrine de la Chine?", Perspectives chinoises, $\mathrm{n}^{\circ} 68$, CEFC (Centre d'études français sur la Chine contemporaine), Hong Kong, novembre-décembre 2001, p. 28-36.

10. C'est-à-dire à l'ouest de la rivière Huangpu (voir carte ci-contre).

11. Située à la frontière de Hong Kong, la ville de Shenzen accueillit au début des années 1980 la première zone économique spéciale de la République populaire de Chine. À ce titre, grand nombre de réformes économiques furent expérimentées sur ce territoire avant d'être généralisées à l'ensemble de la Chine.

12. Hongqiao représente dans ce contexte la zone de développement économique et technique des années 1980, tandis que Minghang constitue une zone de développement industriel.

13. À la fin de l'année 1991, on dénombrait 94 entreprises d'État spécialisées dans la promotion immobilière : 72 d'entre elles étaient chinoises, les 22 restantes étaient des joint venture ou des sociétés étrangères. Durant la seule année 1992, 689 nouvelles entreprises se créèrent.

14. Il s'agit des bureaux de la gestion du sol présents dans chaque arrondissement.

15. Les unités de travail désignent les entreprises d'État ou collectives. Elles devaient encadrer l'ensemble de la société chinoise jusqu'à la réapparition du secteur privé. Elles étaient à ce titre propriétaires d'un parc résidentiel destiné à loger leurs employés.

16. La réforme du logement de 1991 repose essentiellement sur trois points : le lancement du fond d'accumulation commun (gongjijing), l'augmentation des loyers, la commercialisation du parc résidentiel public à prix réduit.

17. Voir V. Laurans, « Logements et confort à Shanghai. L'exemple de wanli, ensemble résidentiel modèle ", Perspectives chinoises, $n^{\circ} 68, \operatorname{CEFC}$ (Centre d'études français sur la Chine contemporaine), Hong Kong, novembre-décembre 2001, p. 37-46.

18. Voir V. Laurans, art. cit.

19. Les étrangers ne sont autorisés à acquérir un logement "pour Chinois » (neixiaofang) qu'à partir d'août 2001. Cette mesure marque la fin de la "politique des waixiaofang ». L'entrée de la Chine dans l'OMC autorise dans un même temps la mondialisation du marché de l'immobilier.

20. Durant l'année 2002 la hausse des prix du logement enregistrée s'échelonne de $20 \%$ à $60 \%$.

21. Voir G. Guiheux, «Immobilier : les autorités à la fois juge et partie », Les Archives de l'actualité, CEFC (Centre d'études français sur la Chine contemporaine), Hong Kong, juin 2005.

22. Les principales orientations du schéma directeur 2000-2020 de Shanghai sont les suivantes: renforcer l'intégration de Pudong; désengorger le centre-ville par une politique de construction massive de logements périurbains et améliorer la desserte des noyaux urbains de banlieue grâce au développement du métro (l'objectif est de pouvoir relier d'ici 2010 les extrémités de la ville, circonscrites par la voie périphérique extérieure, en moins d'une heure. En 2002, trois heures étaient encore nécessaires pour effectuer ce trajet) ; devenir un carrefour commercial grâce à la construction d'un port en eaux profondes, dont l'emplacement est situé à Waigaoqiao, et mettre en service l'aéroport de Pudong.

23. Voir V. Laurans, art. cit.

24. Il s'agit d'habitations construites sur une grande envergure et destinées à une classe moyenne émergente.

25. Le salaire moyen à Shanghai est actuellement évalué à $2000 \mathrm{RMB}$ par personne et par mois. À l'intérieur du périmètre du centre-ville élargi, le prix des logements neufs est quant à lui supérieur ou égal à $9000 \mathrm{RMB}$ le mètre carré.

26. Ce magnat de l'immobilier avait en 2002 fait déplacer les résidents d'un quartier entier de l'arron-dissement de Jin'an dans le cadre d'un projet de rénovation urbaine. Assigné en justice par les habitants dont les indemnités promises n'avaient pas été versées, il fut condamné à une peine -d'emprisonnement de plusieurs années. Cette affaire avait à l'époque défrayé la chronique en raison de la condamnation pour divulgation de secret d'État dont fut ensuite accusé l'avocat 
des plaignants. Zheng Encong avait en fait contacté l'association Amnesty International pour dénoncer les malversations dont étaient victimes ses clients.

27. Maiqili est un lilong (quartier d'habitation typique des anciennes concessions shanghaiennes) faisant l'objet d'un projet de destruction. Début 2005, face au refus obstiné de certains résidents de quitter leur habitation, la société de déplacement en charge des expulsions incendia un logement, causant la mort de deux personnes âgées. Trois des responsables de cette société furent alors condamnés à de très lourdes sanctions (deux peines de mort et un enfermement à perpétuité). Voir Revue économique Caijing, édition du 19 septembre 2005.

28. Le groupe Chengkai était l'une des deux plus importantes sociétés de développement immobilier à Shanghai. Étroitement lié à la municipalité, sa récente dissolution n'est sans doute pas sans rapport avec le limogeage de Cheng Liangyu (le premier secrétaire du Parti communiste de la ville de Shanghai a été suspendu de ses fonctions le 25 septembre 2006) et les enquêtes annoncées par le gouvernement central sur le financement du secteur immobilier shanghaien.

29. En effet, les espaces libérés en centre-ville par les habitants déplacés permettent la construction de logements de standing remplaçant les quartiers populaires d'origine. D'autre part, si les déplacements massifs en banlieue représentent une demande artificielle (les logements sont payés prix coûtant, en partie par les sociétés de déplacement, en partie par les habitants expulsés du centre-ville), ils contribuent à faire monter les prix du parc résidentiel périurbain.

30. Voir les articles de L. Wang dans le Beijing dajun jingji guancha yanjiu zhongxin (Dajun Center for Economics Watch and Studies), www.dajun.com.cn: «Dongqian maodun shi bu pingdeng jiaoyi chansheng de jiegou» [Les contradictions des déplacements sont le résultat d'échanges inéquitables], Pékin, 16 mars 2004 et «Shanghai wei chengshi gaizao suo fuchu de daijia » [Le coût de la rénovation urbaine à Shanghai], Pékin, 15 mars 2004.

31. Concernant le concept de «dominance urbaine", voir H. Lefebvre, La production de l'espace, Paris, Anthropos, 2000.

32. 32 - Cet aspect a fait l'objet de plus amples développements à partir d'exemples précis dans le cadre d'un article précédent. Voir V.Laurans, "Shanghai: l'argument du confort pour déplacer les résidents urbains. Le cas de Jianyeli : projet-pilote de restauration ", Perspectives chinoises, $\mathrm{n}^{\circ} 87$, CEFC (Centre d'études français sur la Chine contemporaine), Hong Kong, janvierfévrier 2005, p. 10-21.

33. Il s'agit d'offrir à chaque ménage la possibilité d'accéder à la propriété d'un ou de plusieurs logements en périphérie urbaine.

\section{INDEX}

Mots-clés : logement urbain, spéculation immobilière, changement social, Shanghai

Keywords : Shanghai, urban housing, real estate speculation, social change 


\section{AUTEUR}

\section{VALÉRIE LAURANS}

Lauréate de la bourse Lavoisier 2005-2006 auprès du Centre d'études français sur la Chine contemporaine de Hong Kong, Valérie Laurans termine un doctorat en aménagement et urbanisme (Institut d'aménagement régional, université Paul Cézanne-Aix/ Marseille 3) qui porte sur les « Chroniques du confort à Shanghai, les transformations de l'habitat ou le jeu social du bien-être domestique de 1996 à 2005 ». Elle a notamment publié dans Perspectives chinoises : « Logements et confort à Shanghai. L'exemple de wanli, ensemble résidentiel modèle » ( $\mathrm{n}^{\circ} 68$, novembre-décembre 2001, p. 37-46) et «Shanghai : l'argument du confort pour déplacer les résidents urbains. Le cas de Jianyeli : projet-pilote de restauration » ( $n^{\circ} 87$, janvier-février 2005, p. 10-21). lauransv@gmail.com 